\title{
Real-time PCR-based detection of Bordetella pertussis and Bordetella parapertussis in an Irish paediatric population
}

\author{
Correspondence \\ Juanita A. Grogan \\ juanita.grogan@olchc.ie
}

Received 7 January 2011

Accepted 3 March 2011

\author{
Juanita A. Grogan, ${ }^{1,2}$ Catriona Logan,, ${ }^{1,2}$ John O'Leary, ${ }^{2,3}$ Rebecca Rush ${ }^{1}$ \\ and Niamh O'Sullivan ${ }^{1,2}$ \\ ${ }^{1}$ Department of Microbiology, Our Lady's Children's Hospital, Dublin, Ireland \\ ${ }^{2}$ Department of Pathology, The Coombe Women and Infants University Hospital, Dublin Ireland \\ ${ }^{3}$ Trinity College, Dublin, Ireland
}

\begin{abstract}
Novel real-time PCR assays targeting the Bordetella pertussis insertion sequence IS481, the toxin promoter region and Bordetella parapertussis insertion sequence IS 1001 were designed. PCR assays were capable of detecting $\leqslant 10$ copies of target DNA per reaction, with an amplification efficiency of $\geqslant 90 \%$. From September 2003 to December 2009, per-nasal swabs and nasopharyngeal aspirates submitted for $B$. pertussis culture from patients $\leqslant 1$ month to $>15$ years of age were examined by real-time PCR. Among 1324 patients, 76 (5.7\%) were $B$. pertussis culture positive and $145(10.95 \%)$ were $B$. pertussis PCR positive. Of the $B$. pertussis PCR-positive patients, $117(81 \%)$ were aged 6 months or less. A total of 1548 samples were examined, of which 87 (5.6\%) were culture positive for $B$. pertussis and $169(10.92 \%)$ were $B$. pertussis $\mathrm{PCR}$ positive. All culture-positive samples were $\mathrm{PCR}$ positive. Seven specimens ( $0.5 \%)$ were $B$. parapertussis culture positive and $10(0.8 \%)$ were $B$. parapertussis PCR positive, with all culture-positive samples yielding PCR-positive results. A review of patient laboratory records showed that of the 1324 patients tested for pertussis $555(42 \%)$ had samples referred for respiratory syncytial virus (RSV) testing and 165 (30\%) were positive, as compared to $19.4 \%$ of the total $\mathbf{5 7 1 9}$ patients tested for RSV in this period. Analysis of the age distribution of RSVpositive patients identified that $129(78 \%)$ were aged 6 months or less, similar to the incidence observed for pertussis in that patient age group. In conclusion, the introduction of the real-time PCR assays for the routine detection of $B$. pertussis resulted in a $91 \%$ increase in the detection of the organism as compared to microbiological culture. The incidence of infection with $B$. parapertussis is low while the incidence of RSV infection in infants suspected of having pertussis is high, with a similar age distribution to $B$. pertussis infection.
\end{abstract}

\section{INTRODUCTION}

Our Lady's Children's Hospital in Dublin is the largest specialist paediatric hospital in the Republic of Ireland and provides secondary care locally and tertiary care nationally. Annually the Microbiology Laboratory receives in excess of 200 requests for Bordetella pertussis culture. The isolation of B. pertussis by culture is insensitive and is affected by a number of factors, including patient age, vaccination status, duration of illness, sample quality, swab type, transport medium and culture conditions (Mattoo \& Cherry, 2005; Lievano et al., 2002).

Pertussis may also be diagnosed by the demonstration of a specific antibody response to pertussis toxin, where the demonstration of a single high value of IgG or IgA is

Abbreviations: RSV, respiratory syncytial virus. diagnostic of a recent or active infection (André et al., 2008; de Melker et al., 2000). The use of a single 'high-titre' serum serology and PCR targeting the IS481 insertion sequence are considered the most efficient diagnostic tools for pertussis in children $>7$ years, adolescents and adults (André et al., 2008). However, the use of serology for pertussis diagnosis in younger children is less useful due to recent vaccination, and the lack of pertussis toxin antibody response in up to $24 \%$ of this age group (Stehr et al., 1998). Diagnostic serology cannot be validly interpreted for one year post-vaccination with acellular pertussis vaccines (Guiso et al., 2011).

It has been reported that pertussis is underdiagnosed in the Republic of Ireland due to the lack of diagnostic tests other than culture, so that diagnosis is based on symptoms (Shahdadpuri et al., 2004). The purpose of this study was 
to develop a real-time PCR assay for the detection of pertussis and to examine the age distribution of the disease in a paediatric population. The multi-copy IS481 is the most popular PCR target used to detect B. pertussis (Muyldermans et al., 2005). As the IS481 sequence occurs in Bordetella holmesii (Reischl et al., 2001) and also in some strains of $B$. bronchiseptica and B. parapertussis a confirmatory PCR using an alternative gene target is required for specific detection of the organism. B. parapertussis may be a cause of infection indistinguishable from pertussis, and a PCR assay targeting this organism was designed as part of this study.

Pertussis in infants frequently presents with apnoea (Mattoo \& Cherry, 2005). Life-threatening apnoea may also occur in very young infants with viral respiratory infection. Respiratory syncytial virus (RSV) is the most common cause of viral lower respiratory tract infection in infants and young children (van Woensel et al., 2003). The difficulty of distinguishing between the two infections is confounded by the fact that the age distributions of RSV infection and $B$. pertussis infection are thought to be very similar. This study also aimed to examine the prevalence of RSV in patients who had clinical samples submitted to the laboratory for pertussis screening.

\section{METHODS}

Primer and probe design. DNA sequences from target genes were retrieved from GenBank (http://www.ncbi.nlm.nih.gov) and aligned using the CLUSTAL W (1.82) multiple sequence alignment packages (http://www.ebi.ac.uk). The GenBank accession numbers of the sequences used for $B$. pertussis IS481 real-time PCR assay design are M28220, M22031, L26973, S66937 and S66929. GenBank accession numbers AF157332-AF157344 were used for B. pertussis toxin promoter real-time PCR assay design. The GenBank accession numbers of $B$. bronchiseptica toxin promoter sequences used for sequence comparison are AY568397-AY568409 and AF157362-AF157364. The GenBank accession numbers of $B$. parapertussis toxin promoter sequences retrieved for sequence comparisons are AF157348AF157361. The GenBank accession number of the B. parapertussis IS1001 sequence used for real-time PCR assay design is X66858.

Sequence areas for primer and probe design were selected on the basis of intra-species homology and inter-species heterogeneity. Primer Express software (Version 2.0 Applied Biosystems) was used to design the primers and MGBNFQ (minor groove binder/non-fluorescent quencher) probes (Table 1). All primers and probes were synthesized by Applied Biosystems.

Preparation of bacterial genomic DNA. Genomic DNA of $B$. pertussis ATCC 8467, B. parapertussis ATCC 15311, B. holmesii ATCC 51541 and B. bronchiseptica ATCC 19395 was isolated using the QIAamp DNA mini kit (Qiagen) (without an RNase A step) according to the manufacturer's instructions. DNA was quantified by spectrophotometry at $260 \mathrm{~nm}$. The specificity and sensitivity of assays was examined using 10-fold serial dilutions (from $18000 \mathrm{pg}$ to $0.18 \mathrm{pg}$ ) of the bacterial genomic DNA samples. Genomic DNA was also used as template for the preparation of positive control plasmid DNA standards.

To further test the specificity of the assays genomic DNA was isolated from the following bacteria using the QIAamp DNA mini kit:
Bordetella petrii NCTC 13317, B. bronchiseptica NCTC 10539, B. avium NCTC 12033, B. ansorpii NCTC 13364, B. pertussis NCTC 10739, B. pertussis NCTC 13251, B. trematum NCTC 13203, B. hinzii NCTC 13199, B. holmesii ATCC 51541, B. parapertussis NCTC 15311, Pseudomonas aeruginosa ATCC 27853, Escherichia coli ATCC 25922, E. coli ATCC 35218, Staphylococcus aureus ATCC 25923, Staph. aureus ATCC 43300, Staph. aureus ATCC 29213, Haemophilus influenzae ATCC 10211, H. influenzae (wild-type), Klebsiella pneumoniae ATCC 700603, Yersinia enterocolitica ATCC 27729, Proteus mirabilis ATCC 43071, P. mirabilis ATCC 12453, Streptococcus pyogenes ATCC 19615, Strep. agalactiae ATCC 27956, Strep. salivarius ATCC 13419, Strep. pneumoniae ATCC 6305, Moraxella catarrhalis ATCC 25238, Enterococcus faecalis ATCC 51299, Ent. gallinarium ATCC 700425, Ent. cassiliflavius ATCC 700327, Corynebacterium pseudodiphthericum ATCC 10700, Bacteroides fragilis ATCC 25285, Clostridium perfringens ATCC 13124 and Fusobacterium necrophorum ATCC 25286. Extracted DNA was quantified spectrophotometrically and $2 \mathrm{ng}$ of the genomic DNA per reaction well was used as template. All bacterial isolates were tested in duplicate wells in the B. pertussis IS481, B. pertussis toxin promoter and B. parapertussis IS1001 assays. To verify that all extracted DNA was amplifiable, the extracted genomic DNA from all bacterial isolates was tested for the presence of the $16 \mathrm{~S}$ rDNA. Primers $(50 \mathrm{nM})$ as described by Mohammadi et al. (2005) and a FAMlabelled MGB probe (CGTATTACCGCGGCTG, $100 \mathrm{nM}$ ) were used to target the $16 \mathrm{~S}$ rDNA in duplicate real-time PCRs.

Preparation of plasmid DNA standards to determine PCR amplification efficiency and assay sensitivity. PCR primer sets targeting DNA sequences external to the real-time PCR target amplicons were designed (Table 2) and used to prepare positivecontrol plasmid DNA standards for each of the real-time PCR assays. Solution-phase PCRs in a final volume of $50 \mu$ l contained $15 \mathrm{mM}$ Tris/HCl, pH 8.0, $50 \mathrm{mM} \mathrm{KCl,} 15 \%$ glycerol, $0.8 \mu \mathrm{M}$ external primers, $200 \mu \mathrm{M}$ (each) dNTP, $2-4 \mathrm{mM} \mathrm{MgCl}_{2}, 2.5$ units AmpliTaq Gold (Applied Biosystems) and $60 \mathrm{ng}$ template bacterial genomic DNA. Amplification reactions were performed on a Perkin Elmer GeneAmp PCR 2400 thermocycler. Cycling conditions were as follows: pre-denaturation/enzyme activation at $95{ }^{\circ} \mathrm{C}$ for $10 \mathrm{~min}$, followed by 40 cycles of denaturation at $94{ }^{\circ} \mathrm{C}$ for $30 \mathrm{~s}$, annealing at $56{ }^{\circ} \mathrm{C}$ for $30 \mathrm{~s}$; extension at $72{ }^{\circ} \mathrm{C}$ for $1 \mathrm{~min} 30 \mathrm{~s}$, with a final extension at $72{ }^{\circ} \mathrm{C}$ for $10 \mathrm{~min}$. PCR products were cloned using the TOPO TA Cloning system (Invitrogen). Plasmid DNA was isolated using the QIAprep miniprep kit (Qiagen) according to the manufacturer's instructions. The nucleotide base sequences of the plasmid DNA inserts were determined by nucleotide sequencing of both DNA strands (Lark Technologies). The sensitivity, amplification efficiency and linearity range of the real-time PCR assays developed were demonstrated by amplifying duplicate aliquots of 10 -fold serial dilutions $\left(10^{6}-10^{0}\right.$ copies $)$ of the appropriate plasmid DNA standard.

Real-time PCR amplification. The primer and probe concentration for each assay was optimized and concentrations that gave the lowest $C_{\mathrm{t}}$ (threshold cycle) and the highest $\Delta R_{\mathrm{n}}$ (the normalized reporter signal minus the baseline signal established in the first few cycles of the PCR) were selected for routine use (Table 1). Reaction volumes of $25 \mu$ contained $1 \times$ TaqMan Mastermix (containing AmpErase uracil- $N$-glycosylase, with dTTP partially replaced by dUTP), the appropriate forward and reverse primer and MGBNFQ probe at concentrations as detailed in Table 1 , and $4 \mu$ lemplate DNA (plasmid dilution, genomic DNA or extracted clinical sample). Realtime PCRs were performed in duplicate on an ABI Prism 7000 or 7500 sequence detector (Applied Biosystems) using universal thermal cycling conditions: $2 \mathrm{~min}$ at $50{ }^{\circ} \mathrm{C}$ (uracil $\mathrm{N}$-glycosylase step), $10 \mathrm{~min}$ at $95{ }^{\circ} \mathrm{C}$ and 40 cycles of $15 \mathrm{~s}$ at $95{ }^{\circ} \mathrm{C}$ and $1 \mathrm{~min}$ at $60{ }^{\circ} \mathrm{C}$.

Controls to minimize false-negative or -positive results. To minimize the potential of false-negative or false-positive results 
Table 1. Real-time oligonucleotide primers and probes designed and used in this study

\begin{tabular}{|llcc|}
\hline Name & \multicolumn{1}{c|}{ Sequence $\left(\mathbf{5}^{\prime}-\mathbf{3}^{\prime}\right)^{\star}$} & Position $\dagger$ & Concn $(\boldsymbol{\mu M}) \ddagger$ \\
\hline Pertussis IS481 Fwd & CAAGGCCGAACGCTTCAT & $862-879$ & $903-926$ \\
Pertussis IS481 Rev & AGTTCTGGTAGGTGTGAGCGTAAG & $881-895$ & 0.05 \\
Pertussis IS481 probe & 6-FAM-CAGTCGGCCTTGCGT-MGB & $60-81$ & 0.05 \\
Pertussis toxin prom Fwd & TTCGTCGTACAAAACCCTCGA & $104-123$ & 0.05 \\
Pertussis toxin prom Rev & GTTCATGCCGTGTTGGATTG & $83-96$ & 0.60 \\
Pertussis toxin prom probe & 6-FAM-CTTCCGTACATCCC-MGB & $952-968$ & 0.60 \\
Parap IS1001 Fwd & CCGGCTCGACGAATTGC & $1013-1034$ & 0.30 \\
Parap IS1001 Rev & AGAACCAGAGCCGTTTGAGTT & $973-986$ & 0.30 \\
Parap IS1001 probe & 6-FAM-AGCCAACCAGCCGCT-MGB & 0.20 \\
\hline
\end{tabular}

*6-FAM, 6-carboxyfluorescein; MGB, minor groove binder.

$\dagger$ Position of each primer or probe is relative to the numbering of the following gene sequences: accession number M28220 for pertussis IS481; accession number AF157344 for pertussis toxin promoter; and accession number X66858 for parapertussis IS1001.

‡Oligonucleotide concentration used real-time PCR amplification reactions.

(Lievano et al., 2002) a series of real-time PCR amplification controls were incorporated into the protocol for the routine screening of respiratory specimens. All PCR plates contained negative extraction controls, no-template controls, and positive amplification controls (quantified plasmid DNA controls). The inclusion of uracil- $N$ glycosylase decontamination as a pre-amplification step further reduced the possibility of cross-contamination.

Extracted specimens were analysed in duplicate wells for the presence of the human $\beta$-actin gene using a pre-designed assay (Applied Biosystems). The detection of human DNA served as a quality check of clinical specimens, and extracted samples with a $\beta$-actin $C_{\mathrm{t}}>36$ cycles were reported as unsuitable for $B$. pertussis and B. parapertussis PCR. Standard curves prepared with the plasmid DNA controls served as a measure of PCR amplification efficiency and checked that no deterioration had occurred in the primers or probes. Known positive samples were used as positive extraction controls.

Patient samples. All per-nasal swabs and nasopharyngeal aspirates received for pertussis culture between September 2003 and December 2009 were included in the study. Per-nasal swabs that were taken in house were rayon-tipped flexible wire swabs in Amies with charcoal transport medium (Copan).

Bacterial culture of patient samples. Nasopharyngeal aspirates and per-nasal swabs were cultured on charcoal blood agar containing cephalexin $\left(40 \mu \mathrm{g} \mathrm{ml}^{-1}\right)$, chocolate agar and blood agar within $1 \mathrm{~h}$ of receipt in the laboratory. The samples were then inoculated into $1 \mathrm{ml}$ PBS and frozen at $-20{ }^{\circ} \mathrm{C}$ for molecular analysis by real-time PCR. Charcoal agar plates were incubated in a moist atmosphere in air for 5 days at $37^{\circ} \mathrm{C}$. Plates were checked at 3 and 5 days. From November 2007 plates were incubated for 7 days with no additional yield. Suspect colonies were identified by standard procedures (Parton, 2005).

Extraction of DNA from patient samples. DNA was extracted from $0.5 \mathrm{ml}$ of the inoculated PBS using the QIAamp DNA mini kit according to the manufacturer's instructions, or the Qiagen M48 biorobot, the MagAttract DNA Mini M48 kit, and the infectious diseases protocol (QIAsoft M Operating System software Version 2.0; Qiagen). Specimen elution was performed in $100 \mu \mathrm{l}$ of nuclease-free water.

Throat swabs from 20 healthy volunteers were collected, inoculated into PBS and processed in the same way as the clinical samples.

RSV infection. To determine if patients had samples referred for direct immunofluorescence detection of RSV the records of patients who were investigated for pertussis were reviewed on the Laboratory Information System. Only virology requests taken within 1 week of the sample for pertussis culture were included in the analysis. The number tested and the number positive for RSV were determined for each of the following age groups: $\leqslant 1$ month, $2-6$ months, 7 months11 years, $12-15$ years and $>15$ years. The total number of patients

Table 2. Oligonucleotide primers used for the preparation of positive-control DNA plasmid standards

\begin{tabular}{|llcc|}
\hline Name & \multicolumn{1}{c|}{ Sequence $\left(\mathbf{5}^{\prime} \mathbf{3}^{\prime}\right)$} & Position & Amplicon length $(\mathbf{b p})$ \\
\hline Pertussis IS481 Fwd ext & TCAAGGACGCAGTGGCCTACTA & $702-723$ & 342 \\
Pertussis IS481 Rev ext & GGTTGTATTCGTCCAGGTTGAG & $1022-1043$ & 218 \\
Pertussis toxin prom Fwd ext & GAAACCGGCGCCAAGCT & $480-496$ & 445 \\
Pertussis toxin prom Rev ext & CGGACGGTGACCGGTACCAT & $148-167$ & $67-711$ \\
Parapertussis IS1001 Fwd ext & CGCCCAACGCATCAA & $1055-1075$ & \\
Parapertussis IS1001 Rev ext & CCAGTGGTTCCAGGCTTGTCT & \\
\hline
\end{tabular}

${ }^{\star}$ Position of each primer or probe is relative to the numbering of the following gene sequences: accession number M28220 for pertussis IS481; accession number AF157344 for pertussis toxin promoter external reverse, accession number BX640422.1 for pertussis toxin promoter forward external, and accession number X66858 for parapertussis IS1001. 
tested and the number positive for RSV in this period were also determined.

\section{RESULTS}

\section{Real-time PCR sensitivity}

Tenfold serial dilutions of plasmid DNA controls (from $10^{6}$ to $10^{0}$ copies) for each assay yielded standard curves (B. pertussis IS481, slope $=-3.58$ and $y$-intercept $=39.40 ; B$. pertussis toxin promoter, slope $=-3.57$ and $y$-intercept $=39.66 ; B$. parapertussis IS 1001, slope $=-3.575, y$-intercept $=40.82$ ), with strong correlation coefficients $\left(r^{2} \geqslant 0.99\right)$, indicating strong linear relationships over the 7-log range examined. PCR amplification efficiency for each assay was calculated from the slope of the standard curves $\left[\%\right.$ efficiency $\left.=\left(10^{-1 / \text { slope }}-1\right) \times 100\right]$ and ranged from $90 \%$ to $91 \%$. A detection limit of $\leqslant 10$ copies of target DNA per reaction was determined for all PCR assays, indicating high assay sensitivity.

\section{Specificity of PCR assay targeting B. pertussis IS481}

Throat swabs taken from healthy volunteers were negative by real-time PCR targeting B. pertussis IS481. Genomic DNA from B. petrii NCTC 13317, B. bronchiseptica NCTC 10539 and ATCC 19395, B. avium NCTC 12033, B. ansorpii NCTC 13364, B. trematum NCTC 13203, B. hinzii NCTC 13199, B. parapertussis ATCC 15311 and human genomic DNA (Applied Biosystems) showed no cross-reaction with the $B$. pertussis IS481 assay. All dilutions of $B$. pertussis genomic DNA (18000 pg to $0.18 \mathrm{pg}$ ) were detected in the real-time PCR assay for $B$. pertussis IS481. As expected, $B$. holmesii was detected with the assay targeting $B$. pertussis IS481, albeit with much lower sensitivity, $B$. pertussis being detected up to six cycles earlier. None of the non-Bordetella species used to examine the specificity of the assay were detected by real-time PCR.

\section{Specificity of PCR assay targeting the B. pertussis toxin promoter}

Genomic DNA from B. holmesii ATCC 51541, B. petrii NCTC 13317, B. avium NCTC 12033, B. ansorpii NCTC 13364, B. trematum NCTC 13203, B. hinzii NCTC 13199, B. parapertussis ATCC 15311 and human genomic DNA were not detected by real-time PCR assay targeting the $B$. pertussis toxin promoter. Throat swabs from healthy volunteers were also PCR negative for the gene target. All dilutions of $B$. pertussis genomic DNA (18000 pg to $0.18 \mathrm{pg}$ ) were detected in the real-time PCR for B. pertussis toxin promoter PCR. When 2 ng genomic DNA was used as template in the $B$. pertussis toxin promoter assay the genomic DNA was detected 3-8 cycles later than with the IS481 assay, depending on the B. pertussis strain tested, the difference being greatest with B. pertussis NCTC 10739 . None of the non-Bordetella species were detected with the assay.
Due to extensive sequence homology between the $B$. pertussis and $B$. bronchiseptica toxin promoter regions, primers and probes targeting the $B$. pertussis toxin promoter were designed to maximize the difference between the $B$. bronchiseptica and B. pertussis primer and probe annealing regions, with four base-pair differences in the forward primer, two base-pair differences in the probe and three base-pair differences in the reverse primer. A weak PCR amplification was observed with $B$. bronchiseptica genomic DNA as template, with an 8-11 cycle difference in the detection of equivalent dilutions of $B$. bronchiseptica genomic DNA as compared to B. pertussis genomic DNA. The $\Delta R_{\mathrm{n}}$ observed with $B$. bronchiseptica genomic DNA as template was also significantly lower, indicating that the DNA of B. bronchiseptica was detected much less efficiently.

\section{Specificity of PCR assay targeting $B$. parapertussis IS1001}

Genomic DNA from B. petrii NCTC 13317, B. bronchiseptica NCTC 10539, B. bronchiseptica ATCC 19395, B. avium NCTC 12033, B. ansorpii NCTC 13364, B. pertussis NCTC 10739, B. pertussis NCTC 13251, B. pertussis ATCC 8467, B. trematum NCTC 13203, B. hinzii NCTC 13199, B. holmesii ATCC 51541, human genomic DNA, and DNA extracted from throat swabs of 20 healthy volunteers were not detected in the real-time PCR assay targeting B. parapertussis IS 1001. All dilutions of $B$. parapertussis genomic DNA (18000 pg to $0.18 \mathrm{pg}$ ) were detected in the real-time PCR B. parapertussis assay targeting IS1001. None of the non-Bordetella species were detected with the B. parapertussis IS1001 assay.

\section{Interpretation of patient sample PCR results}

DNA extracted from all clinical samples was tested in duplicate wells for four PCR targets: $\beta$-actin human DNA, B. pertussis IS481, B. pertussis toxin promoter and $B$. parapertussis IS1001. Specimens were reported PCR positive for B. pertussis only when both $B$. pertussis PCR targets (IS481 and toxin promoter) were positive in both real-time PCR wells. A positive IS481 PCR $\left(C_{\mathrm{t}}<36\right)$ and a negative toxin promoter PCR was considered to be evidence of the presence of $B$. holmesii. A positive IS481 PCR with a $C_{\mathrm{t}}>36$ and a negative toxin promoter PCR was considered indicative of the possible presence of $B$. holmesii or a weak positive reaction for $B$. pertussis as the IS481 assay is for a multi-copy gene and the toxin promoter is a single-copy gene. A positive reaction in the toxin promoter PCR only may be indicative of the presence of $B$. bronchiseptica. Specimens were reported positive for $B$. parapertussis when both duplicate PCR wells tested positive for the B. parapertussis IS1001 target by real-time PCR.

\section{Patient sample results}

In total 1548 samples from 1324 patients were tested for the presence of $B$. pertussis and B. parapertussis. A total of 145 B. pertussis PCR-positive patients and 169 B. pertussis 
Table 3. Age distribution of pertussis detected by real-time PCR and culture

\begin{tabular}{|c|c|c|c|c|c|}
\hline \multirow[t]{2}{*}{ Age } & \multirow[t]{2}{*}{ Total patients } & \multicolumn{2}{|c|}{ B. pertussis } & \multicolumn{2}{|c|}{ B. parapertussis } \\
\hline & & PCR positive (\%) & Culture positive (\%) & PCR positive (\%) & Culture positive (\%) \\
\hline 2-6 months & 441 & $68(15.4)$ & $33(7.5)$ & 2 & 2 \\
\hline 7 months -11 years & 503 & $27(5.4)$ & $13(2.6)$ & 8 & 5 \\
\hline $12-15$ years & 24 & 1 & 1 & 0 & 0 \\
\hline Total & 1324 & $145(10.95)$ & $76(5.7)$ & $10(0.8)$ & $7(0.5)$ \\
\hline
\end{tabular}

PCR-positive samples were identified. Six samples were observed to have a mean $C_{\mathrm{t}}>36$ in the B. pertussis IS481 assay only, with no observable amplification with the $B$. pertussis promoter assay, and were reported as equivocal. A single sample produced a real-time PCR-positive result in the B. pertussis promoter assay only, with no observable amplification with the IS481 assay. Consistent with the criteria determined for reporting $B$. pertussis PCR results, which require that both $B$. pertussis targets be positive in duplicate wells for a specimen to be considered PCR-positive for $B$. pertussis, this sample was reported as negative for $B$. pertussis. As the toxin promoter PCR may test positive for $B$. bronchiseptica, infection with this organism cannot be ruled out, although only normal flora was isolated in culture.

A comparison of the result of real-time PCR detection and microbiological culture identified that $48.5 \%(82 / 169)$ of PCR-positive samples and $47 \%(69 / 145)$ of PCR-positive patients were culture negative for $B$. pertussis. All culturepositive samples were positive by PCR. The age distribution of patients and of positive specimens is detailed in Table 3. Of the B. pertussis PCR-positive samples 29 were nasopharyngeal aspirates. Of these 29 PCR-positive nasopharyngeal samples only 10 were culture positive.

Among the 1324 patients and 1548 samples examined in this study, 7 specimens were culture positive for B. parapertussis while 10 specimens were positive for $B$. parapertussis by realtime PCR. All seven of the culture-positive samples were PCR positive for $B$. parapertussis. The three specimens that were negative by culture had $C_{t}$ values of $>37$ cycles, indicating the presence of very small quantities of the genomic DNA target in extracted clinical specimens.
A total of 27 samples were reported as unsuitable as the $C_{t}$ for $\beta$-actin was $>36$. These samples also tested negative for the presence of $B$. pertussis and B. parapertussis.

\section{RSV results}

Among 1324 patients tested for pertussis, 555 (42\%) had samples referred to the National Virus Reference Laboratory for RSV testing and 165 (30\%) of those tested were positive. Analysis of the age distribution of RSV-positive patients showed that $129(78 \%)$ were aged 6 months or less and $62(38 \%)$ were aged 1 month or less (Table 4$)$. Only 42 B. pertussis PCR-positive patients were tested for RSV and of those 2 were positive. None of the B. parapertussispositive patients were tested for RSV.

The total number of samples referred from our laboratory to the NVRL for RSV testing from September 2003 to December 2009 was 5719, and 1108 (19.4\%) of these were positive. This indicates a higher incidence of RSV in those patients suspected of having pertussis. Analysis of RSVpositive patients showed that $70 \%$ were aged 6 months or less while $54 \%$ of the total requests for RSV were from patients 6 months or less. While no seasonal distribution was evident for pertussis, $78 \%$ of RSV positives were in the winter months, November to January.

\section{DISCUSSION}

This work describes the development of real-time PCR assays for the routine screening of paediatric respiratory specimens for $B$. pertussis and B. parapertussis. The

Table 4. RSV requests and positives

\begin{tabular}{|lcccc|}
\hline Age & $\begin{array}{c}\text { Total RSV } \\
\text { requests }\end{array}$ & $\begin{array}{c}\text { Total RSV } \\
\text { positive (\%) }\end{array}$ & $\begin{array}{c}\text { RSV and pertussis } \\
\text { tested patients }\end{array}$ & $\begin{array}{c}\text { RSV-positive pertussis } \\
\text { tested patients (\%) }\end{array}$ \\
\hline$\leqslant 1$ month & 1158 & $365(32)$ & 158 & $62(39)$ \\
2-6 months & 1926 & $411(21)$ & 224 & $67(30)$ \\
7 months-11 years & 2501 & $328(13)$ & 165 & $36(22)$ \\
12-15 years & 100 & $4(4)$ & 4 & 0 \\
$>15$ years & 34 & $0(0)$ & 4 & $\mathbf{1 6 5}(\mathbf{3 0})$ \\
Total & $\mathbf{5 7 1 9}$ & $\mathbf{1 1 0 8 ( 1 9 )}$ & $\mathbf{5 5 5}$ & \\
\hline
\end{tabular}


resulting assays are highly sensitive, capable of detecting just one copy of the gene targets per reaction. Consistent with the 'consensus recommendations' for use of PCR in the diagnosis of pertussis (Meade \& Bollen, 1994), two assays were designed for the detection of $B$. pertussis, with clinical samples only reported as positive for $B$. pertussis when duplicate wells for both gene targets were detected.

$B$. holmesii contains the IS481 insertion sequence (Reischl et al., 2001) and as expected, genomic DNA of B. holmesii was detected with the assay targeting B. pertussis IS481, albeit with much lower sensitivity. Mazengia et al. (2000) have reported the isolation of $B$. holmesii from patients with pertussis-like symptoms; however, $B$. holmesii was not detected in nasopharyngeal swabs from Finnish and Dutch patients with suspected pertussis (Antila et al., 2006).

The PCR assay targeting the pertussis toxin promoter was designed to fulfil the 'consensus recommendations' for use of PCR in the diagnosis of pertussis (Meade \& Bollen, 1994), facilitating the differentiation of B. pertussis and $B$. holmesii in samples positive for the IS481 target. PCR assays targeting the B. pertussis toxin promoter have been reported to be consistently less sensitive than PCR assays for the multi-copy pertussis IS481 target (Riffelmann et al., 2005). Consistent with these findings, serial dilutions of genomic DNA tested in this study, using both PCR assays, identified a 3-8 cycle difference across the dilution series, with the PCR assay targeting the IS481 becoming positive first. Significant levels of sequence homology are observed between the $B$. pertussis and $B$. bronchiseptica toxin promoter gene sequences. Primers and probes targeting the $B$. pertussis toxin promoter were designed maximizing the difference between the $B$. bronchiseptica and $B$. pertussis primer and probe annealing regions. The resulting assay detects $B$. bronchiseptica, albeit with much lower sensitivity.

Thus clinical samples containing B. bronchiseptica or $B$. holmesii may yield positive results with the $B$. pertussis toxin promoter or B. pertussis IS481 respectively. However, this does not in any way compromise the detection of $B$. pertussis, which requires the detection of both gene targets for the reporting of a positive result.

The real-time PCR assays developed in this study resulted in a $94 \%$ increase in the detection rate in samples containing B. pertussis when compared to culture and a $91 \%$ increase in the detection of positive patients. It is noteworthy however that per-nasal swabs were first cultured on charcoal chocolate and blood agar and therefore quite a lot of original material would have been removed from the swab before the PBS was inoculated. Of the 29 PCR-positive nasopharyngeal samples only 10 were culture positive. The real-time PCR thus resulted in a $290 \%$ increase in the detection rate in these samples, where equivalent amounts of material were processed for PCR and culture. Kösters et al. (2001) reported that $8 \%$ of symptomatic patients were culture positive while $30.4 \%$ were PCR positive. Chan et al. (2002) reported a PCR detection rate of $17 \%$ and culture detection rate of only $1.7 \%$. In this study, $5.7 \%$ of patients were culture positive while the detection rate by PCR was $10.95 \%$. Fry et al. (2009) reported that $18.9 \%$ of samples tested using the same targets but different assays were positive by PCR. In their study samples were limited to infants $\leqslant 6$ months of age that had been admitted to a paediatric intensive care unit, while in our study $41 \%$ were $>6$ months old and all samples, regardless of source, were included.

The factors affecting culture results include age, stage of illness, specimen quality and transport, culture conditions, prior pertussis vaccination, concurrent antibiotic therapy, and time elapsed since cough onset (Mattoo \& Cherry, 2005; Lievano et al., 2002). Culture has previously been reported to be most sensitive in young unvaccinated infants with short duration of symptoms and lowest for adolescents and adults (Wirsing von König et al., 2002). In our study $81 \%$ of patients that were positive for PCR were infants $\leqslant 6$ months of age, and $34 \%$ were $<1$ month old. The incidence of $B$. parapertussis in this patient group was found to be low, at $<0.8 \%$.

The majority of PCR-positive samples were from patients aged $<6$ months, and patients $\leqslant 1$ month old accounted for $34 \%$ (49) of the total PCR-positive patients (Table 3). It has been reported that the incidence of pertussis in adults and older children is increasing: Celentano et al. (2005) reported a $115 \%$ increase in infection in individuals $>14$ years of age in a 5-year study of cases in Western Europe from 1998 to 2002.

The epidemiology of pertussis has recently been studied and reviewed (Cherry, 2005; Mattoo \& Cherry, 2005). Vaccine-induced immunity after three doses lasts for at least 5 years, after which there is a gradual decrease in immunity (Riffelmann et al., 2008). Mooi et al. (2009) reported $B$. pertussis strains with increased toxin production associated with pertussis resurgence. $B$. pertussis infection is the cause of $13-20 \%$ of prolonged cough in adults and adolescents (Cherry, 2005) and often goes unrecognized, providing a reservoir of infection for unvaccinated or incompletely vaccinated infants. Most cases in this study were unvaccinated or incompletely vaccinated infants. In Ireland, in accordance with the current national immunization schedule, pertussis vaccination is given as part of the 5-in-1 Diphtheria, Tetanus, acellular Pertussis/Haemophilus influenzae type $\mathrm{b} /$ Inactivated Polio vaccine (DTaP). The primary course is given at 2, 4 and 6 months, with a booster dose at 4-5 years (Shahdadpuri et al., 2004).

In a carefully controlled study, an acellular vaccine was shown to be protective and safe for use in adults and adolescents (Ward et al., 2005) and the Global Pertussis Initiative Group has recommended the use of booster immunization in adolescents (Plotkin, 2005). In Ireland, a booster dose for adolescents has been recommended since 2008 by the National Immunization Programme, but this has not been implemented. Most adults in Ireland would therefore have little immunity to pertussis. In France and 
Germany DTaP is now given to adolescents between ages 11-13 and 14-16 respectively. In Australia a fourth dose of vaccine is given in mid-adolescence, instead of at 18 months of age (Halperin, 2005).

Pertussis infection may be confused with viral respiratory infection. The initial presentation in infants with pertussis is frequently apnoea (Mattoo \& Cherry, 2005). A fulminant course of pertussis may develop in very young incompletely immunized infants. Fulminant pertussis is characterized by the development of pneumonia that rapidly evolves to respiratory failure requiring cardiovascular support (Sawal et al., 2009). Life-threatening apnoea may occur in very young infants with viral respiratory infection.

RSV is the most common cause of viral lower respiratory tract infection in infants and young children (van Woensel et al., 2003). Our results suggest that patients who were suspected of having $B$. pertussis were more likely to have RSV infection. Occurrence of dual infection with RSV and $B$. pertussis was reported previously (Korppi \& Hiltunen, 2007; Cosnes-Lambe et al., 2008; Walsh et al., 2008). Similarly dual infection occurred in two patients in our study; however it is noteworthy that not all pertussispositive patients were investigated for respiratory viruses. The difficulty of distinguishing between the two infections is confounded by the fact that the age distributions of RSV infection and B. pertussis infection are highly similar. RSV infection is observed to be seasonal while B. pertussis infection is not.

In conclusion, this work reports the design, development and application of real-time PCR assays for the rapid, specific and highly sensitive detection of $B$. pertussis and $B$. parapertussis in paediatric clinical specimens. The evidence would suggest that infants and young children suspected of having pertussis should also be screened for RSV. The application of these PCR assays to the routine laboratory repertoire will provide accurate information about the true incidence and epidemiology of pertussis infection in Ireland.

\section{ACKNOWLEDGEMENTS}

The authors wish to thank the staff of the microbiology laboratory at Our Lady's Children's Hospital for technical support and Dr. Norman Fry for helpful advice on the completion of the manuscript.

\section{REFERENCES}

André, P., Caro, V., Njamkepo, E., Wendelboe, A. M., Van Rie, A. \& Guiso, N. (2008). Comparison of serological and real-time PCR assays to diagnose Bordetella pertussis infection in 2007. J Clin Microbiol 46, 1672-1677.

Antila, M., He, Q., de Jong, C., Aarts, I., Verbakel, H., Bruisten, S., Keller, S., Haanperä, M., Mäkinen, J. \& other authors (2006). Bordetella holmesii DNA is not detected in nasopharyngeal swabs from Finnish and Dutch patients with suspected pertussis. $J$ Med Microbiol 55, 1043-1051.
Celentano, L. P., Massari, M., Paramatti, D., Salmaso, S., Tozzi, A. E. \& EUVAC-NET Group (2005). Resurgence of pertussis in Europe. Pediatr Infect Dis J 24, 761-765.

Chan, E. L., Antonishyn, N., McDonald, R., Maksymiw, T., Pieroni, P., Nagle, E. \& Horsman, G. B. (2002). The use of TaqMan PCR assay for detection of Bordetella pertussis infection from clinical specimens. Arch Pathol Lab Med 126, 173-176.

Cherry, J. D. (2005). The epidemiology of pertussis: a comparison of the epidemiology of the disease pertussis with the epidemiology of Bordetella pertussis infection. Pediatrics 115, 1422-1427.

Cosnes-Lambe, C., Raymond, J., Chalumeau, M., Pons-Catalano, C., Moulin, F., de Suremain, N., Reglier-Poupet, H., Lebon, P., Poyart, C. \& Gendrel, D. (2008). Pertussis and respiratory syncytial virus infections. Eur J Pediatr 167, 1017-1019.

de Melker, H. E., Versteegh, F. G. A., Conyn-van Spaendonck, M. A. E., Elvers, L. H., Berbers, G. A. M., van der Zee, A. \& Schellekens, J. F. P. (2000). Specificity and sensitivity of high levels of immunoglobulin $\mathrm{G}$ antibodies against pertussis toxin in a single serum sample for diagnosis of infection with Bordetella pertussis. J Clin Microbiol 38, 800-806.

Fry, N. K., Duncan, J., Wagner, K., Tzivra, O., Doshi, N., Litt, D. J., Crowcroft, N., Miller, E., George, R. C. \& Harrison, T. G. (2009). Role of PCR in the diagnosis of pertussis infection in infants: 5 years' experience of provision of a same-day real-time PCR service in England and Wales from 2002 to 2007. J Med Microbiol 58, 1023 1029.

Guiso, N., Berbers, G., Fry, N. K., He, Q., Riffelmann, M., Wirsing von König, C. H. \& EU Pertstrain Group (2011). What to do and what not to do in serological diagnosis of pertussis: recommendations from EU reference laboratories. Eur J Clin Microbiol Infect Dis 30, 307-312.

Halperin, S. A. (2005). Pertussis - a disease and vaccine for all ages. $N$ Engl J Med 353, 1615-1617.

Korppi, M. \& Hiltunen, J. (2007). Pertussis is common in nonvaccinated infants hospitalized for respiratory syncytial virus infection. Pediatr Infect Dis J 26, 316-318.

Kösters, K., Riffelmann, M. \& Wirsing von König, C. H. (2001). Evaluation of a real-time PCR assay for detection of Bordetella pertussis and B. parapertussis in clinical samples. J Med Microbiol 50, 436-440.

Lievano, F. A., Reynolds, M. A., Waring, A. L., Ackelsberg, J., Bisgard, K. M., Sanden, G. N., Guris, D., Golaz, A., Bopp, D. J. \& other authors (2002). Issues associated with and recommendations for using PCR to detect outbreaks of pertussis. J Clin Microbiol 40, 2801-2805.

Mattoo, S. \& Cherry, J. D. (2005). Molecular pathogenesis, epidemiology, and clinical manifestations of respiratory infections due to Bordetella pertussis and other Bordetella subspecies. Clin Microbiol Rev 18, 326-382.

Mazengia, E., Silva, E. A., Peppe, J. A., Timperi, R. \& George, H. (2000). Recovery of Bordetella holmesii from patients with pertussislike symptoms: use of pulsed-field gel electrophoresis to characterize circulating strains. J Clin Microbiol 38, 2330-2333.

Meade, B. D. \& Bollen, A. (1994). Recommendations for use of the polymerase chain reaction in the diagnosis of Bordetella pertussis infections. J Med Microbiol 41, 51-55.

Mohammadi, T., Pietersz, R. N., Vandenbroucke-Grauls, C. M., Savelkoul, P. H. \& Reesink, H. W. (2005). Detection of bacteria in platelet concentrates: comparison of broad-range real-time $16 \mathrm{~S}$ rDNA polymerase chain reaction and automated culturing. Transfusion 45, 731-736.

Mooi, F. R., van Loo, I. H. M., van Gent, M., He, Q., Bart, M. J., Heuvelman, K. J., de Greeff, S. C., Diavatopoulos, D., Teunis, P. \& other authors (2009). Bordetella pertussis strains with increased toxin 
production associated with pertussis resurgence. Emerg Infect Dis 15, 1206-1213.

Muyldermans, G., Soetens, O., Antoine, M., Bruisten, S., Vincart, B., Doucet-Populaire, F., Fry, N. K., Olcén, P., Scheftel, J. M. \& other authors (2005). External quality assessment for molecular detection of Bordetella pertussis in European laboratories. J Clin Microbiol 43, 30-35.

Parton, R. (2005). Bordetella. In Topley \& Wilson's Microbiology and Microbial Infections, pp. 1786-1817. Edited by S. P. Borriello, P. R. Murray \& G. Funke. London: Edward Arnold.

Plotkin, S. (2005). The global pertussis initiative: process overview. Pediatr Infect Dis J 24 (Suppl.), S7-S9.

Reischl, U., Lehn, N., Sanden, G. N. \& Loeffelholz, M. J. (2001). Realtime PCR assay targeting IS481 of Bordetella pertussis and molecular basis for detecting Bordetella holmesii. J Clin Microbiol 39, 1963-1966.

Riffelmann, M., Wirsing von König, C. H., Caro, V., Guiso, N. \& Pertussis PCR Consensus Group (2005). Nucleic acid amplification tests for diagnosis of Bordetella infections. J Clin Microbiol 43, 49254929.

Riffelmann, M., Littmann, M., Hülße, C., Hellenbrand, W. \& Wirsing von König, C. H. (2008). Pertussis: not only a disease of childhood. Dtsch Arztebl Int 105, 623-628.

Sawal, M., Cohen, M., Irazuzta, J. E., Kumar, R., Kirton, C., Brundler, M.-A., Evans, C. A., Wilson, J. A., Raffeeq, P. \& other authors (2009).
Fulminant pertussis: a multi-center study with new insights into the clinico-pathological mechanisms. Pediatr Pulmonol 44, 970-980.

Shahdadpuri, R. I., Nicholson, A. J., Bedford, D. \& O'Flanagan, D. (2004). Pertussis - going, going, but not gone: thirteen year trends in the incidence of pertussis in the Republic of Ireland and North Eastern Health Board. Ir Med J 97, 44-46.

Stehr, K., Cherry, J. D., Heininger, U., Schmitt-Grohé, S., Überall, M., Laussucq, S., Eckhardt, T., Meyer, M., Engelhardt, R. \& other authors (1998). A comparative efficacy trial in Germany in infants who received either the Lederle/Takeda acellular pertussis component DTP (DTaP) vaccine, the Lederle whole-cell component DTP vaccine, or DT vaccine. Pediatrics 101, 1-11.

van Woensel, J. B. M., van Aalderen, W. M. C. \& Kimpen, J. L. L. (2003). Viral lower respiratory tract infection in infants and young children. BMJ 327, 36-40.

Walsh, P., Overmeyer, C., Kimmel, L., Feola, M., Pusavat, J., Nguyen, T. A., Kuan, S., Emery, K., Rosengreen, M. \& other authors (2008). Prevalence of Bordetella pertussis and Bordetella parapertussis in samples submitted for RSV screening. West J Emerg Med 9, 135-140.

Ward, J. I., Cherry, J. D., Chang, S. J., Partridge, S., Lee, H., Treanor, J., Greenberg, D. P., Keitel, W., Barenkamp, S. \& other authors (2005). Efficacy of an acellular pertussis vaccine among adolescents and adults. N Engl J Med 353, 1555-1563.

Wirsing von König, C. H., Halperin, S., Riffelmann, M. \& Guiso, N. (2002). Pertussis of adults and infants. Lancet Infect Dis 2, 744-750. 